\title{
DISJOINTNESS IN TRANSFORMATION GROUPS
}

\author{
HARVEY B. KEYNES ${ }^{1}$
}

\begin{abstract}
In this paper, we shall be concerned with the question of what conditions on minimal transformation groups will guarantee that they are disjoint. Generalizing a result of $I$. Bronštein about lifting of minimality through group extensions to associated bitransformation groups, we prove that in a large class of transformation groups, disjointness is equivalent to disjointness of their maximal equicontinuous factors. In the abelian case, this means that disjointness is equivalent to no common factor in the class of flows discussed.
\end{abstract}

1. Introduction. Several authors ([2], [4], and [5]) have been concerned with the problem of when disjointness in abelian minimal transformation groups is equivalent to no common factors. The best result is found in [5, Theorem 12], where it is shown that if two metric transformation groups are both PD transformation groups (built by successive proximal and distal extensions), then they are disjoint iff they have no common factors. In this paper, we show that if only one transformation group is a PD transformation group, then the same result holds. Moreover, we can relax the metric assumption completely on one transformation group, and partially on the other. The main idea involved is lifting minimality through a special type of group extension called an associated bitransformation group. Finally, an application is given in the point-distal case.

All transformation groups in this paper will have compact $T_{2}$ phase spaces and discrete acting groups. We recall that two minimal transformation groups $(X, T),(Y, T)$ are disjoint if $(X \times Y, T)$ is minimal. By a bitransformation group $(G, X, T)$, we shall mean that $G$ is a freely-acting compact topological group of automorphisms of $(X, T)$ acting in a jointly-continuous fashion on $X$. We shall denote the equicontinuous and distal structure relations by $S(X)$ and $D(X)$ respectively; thus $(X / S(X), T)$ and $(X / D(X), T)$ are the maximal equicontinuous and distal factors of $(X, T)$ respectively. The notation $\varphi:(X, T) \rightarrow(Y, T)$ means that $\varphi$ is a

Received by the editors January 8, 1972.

AMS 1969 subject classifications. Primary 5480, 5482.

Key words and phrases. Minimal transformation group, disjointness, bitransformation group, distal extensions, proximal extensions.

'Research supported by NSF Grant GP-29321. 
homomorphism from $(X, T)$ onto $(Y, T)$, or $(Y, T)$ is a factor of $(X, T)$. If $\varphi(x)=\varphi\left(x_{1}\right)$ implies $x$ and $x_{1}$ are distal (resp. proximal), then $\varphi$ is called a distal (resp. proximal) extension. A related type of extension is given as follows: set $R(X, Y)=\left\{\left(x, x_{1}\right) \mid \varphi(x)=\varphi\left(x_{1}\right)\right\}$, and define $Q(X, Y)=$ $\bigcap\{\operatorname{cls}(R(X, Y) \cap \alpha T) \mid \alpha$ index on $X\}$. Then $\varphi$ is an almost periodic extension if $Q(X, Y)=\Delta$, the diagonal. We shall encounter all three types of extensions in this paper.

Finally, it should be noted that R. Ellis has obtained another proof, using the algebraic machinery, of some of these results.

2. Associated bitransformation groups. Our major goal will be to lift disjointness through certain types of extensions which are generalizations of group extensions. We shall need to generalize the following result of Bronštein [1] to these extensions:

THEOREM (2.1). Let $(G, X, T)$ be a bitransformation group. Then $(X, T)$ is minimal iff $(X / G, T)$ and $(X / S(X), T)$ are minimal.

We now define the necessary type of extension.

Definition (2.2). Let $(G, X, T)$ be a bitransformation group with $G$ compact and acting jointly continuously on $X$. Let $M$ be a compact $T_{2}$ space, and suppose that $(G, M)$ is a left transformation group. Form the bitransformation group $(G, X \times M, T)$ where $g(x, m)=(g x, g m)$, and $(x, m) t=(x t, m)$. Then $(G, X \times M, T)$ is the $M$-associated bitransformation group.

Since $G$ is compact, we have that the orbit space $X \times M / G$ is compact, $T_{2}$. Also, if $M$ is a point, then we recover (isomorphically) $(G, X, T)$.

LEMMA (2.3). Let $(G, X \times M, T)$ be an $M$-associated bitransformation group. Suppose that $(X, T)$ is minimal, and $(G, M)$ is transitive. Then $(X \times M / G, T)$ is minimal.

Proof. Let $\langle x, m\rangle,\left\langle x_{1}, m_{1}\right\rangle \in X \times M / G$. Since $G$ acts transitively on $M$, we have that $m_{1}=g^{-1} m$ for some $g \in G$. Thus, $\left\langle x_{1}, m_{1}\right\rangle=\left\langle g^{-1}\left(g x_{1}\right), g^{-1} m\right\rangle=$ $\left\langle g x_{1}, m\right\rangle$. Since $(X, T)$ is minimal, we have that $x t_{n} \rightarrow g x_{1}$ for some net $\left(t_{n}\right) \in T$. Then $\langle x, m\rangle t_{n}=\left\langle x t_{n}, m\right\rangle \rightarrow\left\langle g x_{1}, m\right\rangle$, as desired.

The next result is the desired generalization $((X, T) \simeq(X \times G / G, T)$ via the map $q(x)=\langle x, e\rangle)$.

THEOREM (2.4). Let $(G, X \times M, T)$ be an $M$-associated bitransformation group. Set $Y=X \times M / G$. Then $(Y, T)$ is minimal iff $(X / G, T)$, $(Y / S(Y), T)$ are minimal.

Proof. Since pr: $(G, X \times M, T) \rightarrow(G, X, T)$ induces $\varphi:(Y, T) \rightarrow(X / G, T)$ it follows that $(Y, T)$ being minimal yields that $(X / G, T),(Y / S(Y), T)$ are minimal. 
Conversely, suppose $(X / G, T)$ and $(Y / S(Y), T)$ are minimal. Since $(X, T)$ is a distal extension of $(X / G, T),(X, T)$ is pointwise almost periodic. Let $Z$ be a minimal subset of $X$. Set $K=\{g \mid g \in G$ and $g(Z)=Z\}$. Then $K$ is a closed subgroup of $G$, and we can form the associated bitransformation group $(K, Z \times M, T)$. Let $\pi: Z \times M \rightarrow Z \times M / K, \pi_{1}: X \times M \rightarrow X \times M / G$ be the canonical projections. Define $\varphi: Z \times M / K \rightarrow X \times M / G$ by $\varphi(\pi(z, m))=\pi_{1}(z, m)$. To see that $\varphi$ is onto, let $x \in X, m \in M$. Since $(X / G, T)$ is minimal, $g x \in Z$ for some $g \in G$. Then $\varphi(\pi(g x, g m))=$ $\pi_{1}(g x, g m)=\pi_{1}(x, m)$. Next, suppose $\pi_{1}(z, m)=\pi_{1}\left(z_{1}, m_{1}\right)$. Then $g z=z_{1}$, $g m=m_{1}$ for some $g \in G$. Since $z, z_{1} \in Z, g Z=Z$ and $g \in K$. Thus, $\pi(z, m)=\pi\left(z_{1}, m_{1}\right)$, and $\varphi$ is one-to-one. Finally, if $\pi\left(z_{n}, m_{n}\right) \rightarrow \pi(z, m)$, then for some net $\left(k_{n}\right) \in K, k_{n}\left(z_{n}, m_{n}\right) \rightarrow(z, m)$, since $\pi$ is open. Hence $\pi_{1}\left(k_{n}\left(z_{n}, m_{n}\right)\right)=\pi_{1}\left(z_{n}, m_{n}\right) \rightarrow \pi_{1}(z, m)$, and $\varphi$ is continuous. Thus,

$$
\varphi:(Z \times M / K, T) \simeq(X \times M / G, T) .
$$

We now consider the transformation group $(M / K, T)$, where $T$ acts trivially. Since in the associated bitransformation group $(K, Z \times M, T)$, $T$ acts only on $Z$, it follows that we have $\rho:(Z \times M / K, T) \rightarrow(M / K, T)$ via the canonical map. Since $(M / K, T)$ is equicontinuous and $(Z \times M / K, T)$ is isomorphic to $(Y, T)$ by the above, then $(M / K, T)$ is a homomorphic image of $(Y / S(Y), T)$ and hence is minimal. By the definition of the action of $T$, this means that $M / K$ is a point. This is equivalent to the fact that $K$ acts transitively on $M$. By (2.3), it follows that $(Z \times M / K, T)=(Y, T)$ is minimal, as desired.

To apply Theorem (2.4), we recall the notion of quasi-separability [4], which is equivalent to sufficiently many metric factors [4, Theorem 2.2]. The generalized Furstenberg structure theorem [2, (15.4)] can then be stated as follows:

THEOREM. Let $(X, T),\left(X_{0}, T\right)$ be minimal transformation groups such that $(X, T)$ is quasi-separable and a distal extension of $\left(X_{0}, T\right)$. Then there exists an ordinal $v$ and families $\left(\left(X_{\alpha}, T\right) \mid \alpha \leqq v\right),\left(\left(Y_{\alpha}, T\right) \mid \alpha \leqq v\right)$ such that:

(1) $\left(X_{v}, T\right) \simeq(X, T)$.

(2) If $\alpha<v$, then $\left(Y_{\alpha+1}, T\right)$ is a group extension of $\left(X_{\alpha}, T\right)$ and $\left(X_{\alpha+1}, T\right)$ is an almost periodic extension of $\left(X_{\alpha}, T\right)$.

(3) If $\alpha<v$, then $\left(Y_{\alpha+1}, T\right) \rightarrow\left(X_{\alpha+1}, T\right) \rightarrow\left(X_{\alpha}, T\right)$.

(4) If $\alpha \leqq v$ is a limit ordinal, then $\left(X_{\alpha}, T\right)=\operatorname{inv} \lim \left(\left(X_{\beta}, T\right) \mid \beta<\alpha\right)$, $\left(Y_{\alpha}, T\right)=\operatorname{inv} \lim \left(\left(Y_{\beta}, T\right) \mid \beta<\alpha\right)$.

The important point to note is that $(X, T)$ is built from $\left(X_{0}, T\right)$ via the family $\left(\left(X_{\alpha}, T\right) \mid \alpha \leqq v\right)$, and not by the group extensions $\left(\left(Y_{\alpha+1}, T\right) \mid \alpha \leqq v\right)$. With almost periodic extensions, we have the following lemma. 
LemMa. Let $(X, T)$ and $(Y, T)$ be minimal transformation groups with $(X, T)$ an almost periodic extension of $(Y, T)$. Then there exist a compact $T_{2}$ group $G$, a closed subgroup $K$, and a bitransformation group $(G, Z, T)$ such that if $(G, Z \times G / K, T)$ is the $G / K$-associated bitransformation group, then $(X, T) \simeq((Z \times G / K) / G, T)$, and $(Y, T) \simeq(Z / G, T)$.

A proof of this lemma can be found in $[2,(12,13)$ and $(12,14)]$, together with the fact that $G$ can be chosen with a compact $T_{2}$ group topology which yields a jointly continuous action on $Z$ [2, (14.11), (14.26)]. In the metric case, almost periodic extensions can be replaced by isometric extensions [2, (1.2) of notes, Chapter 15], and the lemma is implicit in Furstenberg's original paper on distal transformation groups.

3. Disjointness relations. In preparation for the main result, we now state some algebraic preliminaries. We shall be considering minimal transformation groups $(X, T),(Y, T)$ whose associated algebras $\mathscr{A}, \mathscr{B} \subset$ $\mathfrak{U}(u)$ satisfy $\mathscr{A}, \mathscr{B} \subset \mathscr{K}=\{f \in \mathfrak{U}(u) \mid f t \in \mathfrak{X}(u)(t \in T)\}$. This class includes point-distal and abelian transformation groups. It satisfies the property that disjointness is determined by the groups $A, B$ of $\mathscr{A}, \mathscr{B}$, respectively; $(X, T)$ is disjoint from $(Y, T)$ iff $A B=G[2,(18.7)]$. See [3] as a general reference.

We also can define the class $\mathrm{PD}(T)$ of proximal-distal transformation groups for a given discrete group $T$ as follows: Let $\mathscr{B}_{0}=\mathscr{E}$, the universal equicontinuous algebra, $\mathscr{B}_{1}=\mathscr{E}^{*}=\mathscr{D}$, i.e., the maximal distal algebra extension of $\mathscr{E}$, which is $\mathscr{D}$, the universal distal algebra, $B_{2}=\mathfrak{U}(D)$, the maximal proximal algebra extension of $\mathscr{D}$ (here $D$ is the group of $\mathscr{D}$ ). In general, suppose $\mathscr{B}_{\alpha+1}$ is defined. If $\left(\left|\mathscr{B}_{\alpha+1}\right|, T\right) \rightarrow\left(\left|\mathscr{B}_{\alpha}\right|, T\right)$ is a proximal extension, define $\mathscr{B}_{\alpha+2}=\mathscr{B}_{\alpha+1}^{*}$, the maximal distal extension algebra of $\mathscr{B}_{\alpha+1}$, if $\mathscr{B}_{\alpha+1}^{*} \neq \mathscr{B}_{\alpha+1}$. Otherwise, stop the process. If $\left(\left|\mathscr{B}_{\alpha+1}\right|, T\right) \rightarrow\left(\left|\mathscr{B}_{\alpha}\right|, T\right)$ is a distal extension, define $\mathscr{B}_{\alpha+2}=\mathfrak{U}\left(B_{\alpha+1}\right)$, the maximal proximal extension, if $\mathfrak{Y}\left(B_{\alpha+1}\right) \neq \mathscr{B}_{\alpha+1}$. Otherwise, stop the process. At limit ordinals $\gamma$, set $\mathscr{B}_{\gamma}=\left\langle\mathscr{B}_{\alpha}\right| \alpha\langle\gamma\rangle$, the generated subalgebra. Now there exists a minimal ordinal $\alpha_{0}$ with $\operatorname{crd} \alpha_{0} \leqq \operatorname{crd}(\mathscr{C}(\beta T))$ for which the process stops. Define $\operatorname{PD}(T)=\left\{\mathscr{B}_{\alpha} \mid \alpha \leqq x_{0}\right\}$. If $\mathscr{A} \subset \mathfrak{U}(u)$, the maximal PD subalgebra is $\mathscr{A} \cap \mathscr{B}_{\beta}$ where $\beta$ is minimal with respect to $\mathscr{A} \cap \mathscr{B}_{\beta+1}=\mathscr{A} \cap \mathscr{B}_{\beta}$. Finally $\mathscr{A}$ is a PD algebra (and $(|\mathscr{A}|, T)$ a PD transformation group) if $\mathscr{A} \subset \mathscr{B}_{\alpha}$, for some $\alpha$. Thus, $(|\mathscr{A}|, T)$ can be built by a chain of successive distal and proximal extensions starting from its maximal equicontinuous factor.

Finally, we say that $(X, T)$ is totally quasi-separable if $(X, T)$ and every factor are quasi-separable. Clearly, this holds if $X$ is metric. It also holds when $T$ supports a $\sigma$-compact topological group topology under which the action is jointly continuous. For then the associated algebra $\mathscr{A} \subset \mathscr{R}$, the 
bounded right uniformly continuous functions on $T$, and thus so is every subalgebra. Again, see [4].

We show now the following useful lemma.

Lemma (3.1). Let $(X, T),(Y, T)$ be $\mathscr{K}$-transformation groups. Then $(X, T) \perp(Y / S(Y), T)$ iff $(X / S(X), T) \perp(Y \mid S(Y), T)$.

Proof. If $\mathscr{A}, \mathscr{B}$ are associated algebras for $X, Y$, then $(X \mid S(X), T) \perp$ $(Y \mid S(Y), T)$ is equivalent to $(A E)(B E)=G$; see [2, (14.16)]. Now $E$, the group of the universal equicontinuous algebra, is normal in $G$. Hence, $(A E)(B E)=A(B E)=G$. Thus, $(X, T) \perp(Y / S(Y), T)$. The other way is obvious, completing the proof.

Lemma (3.2). Let $(X, T),(Y, T),(Z, T)$ be $\mathscr{K}$-transformation groups such that $(Z, T)$ is a quasi-separable and distal extension of $(Y, T)$ and there exists a homomorphism $\varphi:(Y \mid S(Y), T) \rightarrow(Z / S(Z), T)$. Suppose that $(X, T) \perp(Y, T)$. Then $(X, T) \perp(Z, T)$.

Proof. By the generalized Furstenberg structure theorem, we have a family $\left(\left(Y_{\beta}, T\right) \mid \beta \leqq \beta_{0}\right)$, where $\left(Y_{0}, T\right) \simeq(Y, T), \quad\left(Y_{\beta_{0}}, T\right) \simeq(Z, T)$ and $\left(Y_{\beta+1}, T\right)$ is an almost periodic extension of $\left(Y_{\beta}, T\right)$. By the lemma of $\S 2$, there is an associated bitransformation group yielding $\left(Y_{\beta+1}, T\right) \rightarrow\left(Y_{\beta}, T\right)$.

Fix $\beta$, and assume $(X, T) \perp\left(Y_{\beta}, T\right)$. By letting the group from the associated bitransformation group for $\left(Y_{\beta+1}, T\right) \rightarrow\left(Y_{\beta}, T\right)$ act trivially on $X$, the associated bitransformation group can be extended to yield $\left(X \times Y_{\beta+1}, T\right) \rightarrow\left(X \times Y_{\beta}, T\right)$. Now we have $(Z / S(Z), T) \rightarrow\left(Y_{\beta+1} / S\left(Y_{\beta+1}\right), T\right)$ and $\left(Y_{\beta+1} / S\left(Y_{\beta+1}\right), T\right) \rightarrow(Y / S(Y), T)$. Since we have $\varphi:(Y / S(Y), T) \rightarrow$ $(Z / S(Z), T)$ and the fact that almost periodic minimal sets are coalescent (every endomorphism is an isomorphism), then

$$
(Y \mid S(Y), T) \simeq\left(Y_{\beta+1} / S\left(Y_{-\beta+1}\right), T\right) .
$$

As we are dealing with $\mathscr{K}$-transformation groups, we have that $S\left(X \times Y_{\beta+1}\right) \simeq S(X) \times S\left(Y_{\beta+1}\right)$ [3, Remark (3.3)]. Then

$$
\begin{aligned}
\left(X \times Y_{\beta+1} / S\left(X \times Y_{\beta+1}\right), T\right) & \simeq\left(X / S(X) \times Y_{\beta+1} / S\left(Y_{\beta+1}\right), T\right) \\
& \simeq(X / S(X) \times Y / S(Y), T)
\end{aligned}
$$

and is minimal, since $(X \times Y, T)$ is minimal. Since $\left(X \times Y_{\beta}, T\right)$ is minimal by assumption, we have by $(2.4)$ that $\left(X \times Y_{\beta+1}, T\right)$ is minimal.

If $\beta^{\prime}$ is a limit ordinal and $(X, T) \perp\left(Y_{\beta}, T\right)$ for $\beta \leqq \beta^{\prime}$, then $(X, T) \perp$ $\left(Y_{\beta^{\prime}}, T\right): Y_{\beta^{\prime}}=$ inv $\lim _{\beta<\beta^{\prime}} Y_{\beta}$ implies that $X \times Y_{\beta^{\prime}}=$ inv $\lim _{\beta<\beta^{\prime}} X \times Y_{\beta}$. Since $\left(X \times Y_{\beta}, T\right)$ is minimal if $\beta<\beta^{\prime}$, we then have that $\left(X \times Y_{\beta^{\prime}}, T\right)$ is minimal, as desired.

The proof of the lemma is now completed by transfinite induction. 
We now prove the main result

THEOREM (3.3). Let $(X, T)$ and $(Y, T)$ be $\mathscr{K}$-transformation groups such that $(Y, T)$ is a PD transformation group and totally quasi-separable. Then $(X, T) \perp(Y, T)$ iff $(X \mid S(X), T) \perp(Y \mid S(Y), T)$.

Proof. We have that there exists a family $\left(\left(Y_{\gamma}, T\right) \mid \gamma \leqq \gamma_{0}\right)$ with $\left(Y_{0}, T\right) \simeq(Y \mid S(Y), T)$ and the extensions being either proximal or distal, or an inverse limit, and $\left(Y_{\gamma_{0}}, T\right) \simeq(Y, T)$.

Assume that $(X / S(X), T) \perp(Y \mid S(Y), T)$. Then by $(3.1),(X, T) \perp$ $(Y / S(Y), T) \simeq\left(Y_{0}, T\right)$. Next, suppose that $(X, T) \perp\left(Y_{\alpha}, T\right)$. If $\left(Y_{\alpha+1}, T\right)$ is a proximal extension of $\left(Y_{\alpha}, T\right)$, then the fact that $(X, T) \perp\left(Y_{\alpha+1}, T\right)$ is obvious from the groups [2, (18.8)]. If $\left(Y_{\alpha+1}, T\right)$ is a distal extension of $\left(Y_{\alpha}, T\right)$, then we use (3.2) to show that $(X, T) \perp\left(Y_{\alpha+1}, T\right)$. Handling the inverse limit case as in (3.2), we conclude by transfinite induction that $(X, T) \perp(Y, T)$, as desired. This completes the proof, since the other way is trivial.

As a corollary, we now show that disjointness and no common factor are equivalent in the abelian case when one transformation group is PD.

COROLlaRY (3.4). Let $(X, T),(Y, T)$ be minimal transformation groups with $T$ abelian, and $(Y, T)$ a $P D$ transformation group which is totally quasi-separable. Then $(X, T) \perp(Y, T)$ iff $(X, T)$ and $(Y, T)$ have no common factor.

Proof. If $(X, T)$ and $(Y, T)$ have no common factor, then neither do $(X / S(X), T)$ and $(Y / S(Y), T)$. Since $T$ is abelian, this means that $(X / S(X), T) \perp(Y / S(Y), T)$ by $[4$, Theorem (4.7)]. Then $(X, T) \perp(Y, T)$ by (3.3). Since the other way is trivial, the proof is completed.

We now prove a slight generalization to [5, Theorem 14].

COROLlaRY (3.5). Let $(X, T)$ be a totally quasi-separable minimal transformation group, and $(Y, T)$ a $\mathscr{K}$ and $P D$ transformation group which is totally quasi-separable. Then $(X, T) \perp(Y, T)$ iff $(X / S(X), T) \perp(Y \mid S(Y), T)$.

Proof. Suppose $(X / S(X), T) \perp(Y / S(Y), T)$. Since distal transformation groups are always $\mathscr{K}$-transformation groups, we have that $(X / D(X), T) \perp(Y, T)$ by (3.3). Then $(X, T) \perp(Y, T)$ by [5, Lemma 13]. The other way is obvious.

Ellis has recently proved a result which implies among other things that if one starts with a point-distal metric transformation group $(X, T)$ with the distal point coming from $u$, then one can extend the Veech theory to produce a PD metric transformation group $\left(X^{*}, T\right)$ which is almost automorphic over $(X, T)$ and also has $u$ yielding a distal point. This 
means that $\left(X^{*}, T\right)$ is also a $\mathscr{K}$-transformation group. Thus, we have:

COROllary (3.6). Let $(X, T)$ be a point-distal metric transformation group with the distal point coming from $u$, and $(Y, T)$ a $\mathscr{K}$-transformation group. Then $(X, T) \perp(Y, T)$ iff $(X / S(X), T) \perp(Y \mid S(Y), T)$.

Proof. Since $\left(X^{*}, T\right)$ is a proximal extension of $(X, T)$, then

$$
\left(X^{*} / S\left(X^{*}\right), T\right) \simeq(X / S(X), T) .
$$

Thus, $\left(X^{*} / S\left(X^{*}\right), T\right) \perp(Y / S(Y), T)$, and by (3.3), $\left(X^{*}, T\right) \perp(Y, T)$. Since $(X, T)$ is a factor of $\left(X^{*}, T\right)$, we have that $(X, T) \perp(Y, T)$, as desired.

\section{BIBLIOGRAPHY}

1. I. Bronšteĭn, On distal minimal sets, Mat. Issled. 5 (1970). (Russian)

2. R. Ellis, Lectures on topological dynamics, Benjamin, New York, 1969. MR 42 \#2463.

3. R. Ellis and H. Keynes, A characterization of the equicontinuous structure relation, Trans. Amer. Math. Soc. 161 (1971), 171-183.

4. H. Keynes, The structure of weakly mixing minimal transformation groups, Illinois J. Math. 15 (1971), 475-489.

5. R. Peleg, Weak disjointness of transformation groups, Proc. Amer. Math. Soc. 33 (1972), 165-170.

School of Mathematics, University of Minnesota, Minneapolis, Minnesota 55455 